\begin{tabular}{cc} 
Sharif University of Technology \\
Scientia Iranica \\
SCIENTIA & Transactions F: Nanotechnology \\
I RAN I CA & http://scientiairanica.sharif.edu \\
\hline
\end{tabular}

\title{
Numerical study of hydrothermal characteristics in nanofluid using KKL model with Brownian motion
}

\author{
Sh. Rana, M. Nawaz*, and I. Haider Qureshi \\ Department of Applied Mathematics \& Statistics, Institute of Space Technology, Islamabad, 44000, Pakistan.
}

Received 22 December 2017; received in revised form 10 October 2018; accepted 5 January 2019

\section{KEYWORDS}

KKL-model;

Effective diffusion

coefficients;

Entropy generation;

Brownian motion;

Thermal radiation.

\begin{abstract}
Finite Element Method (FEM) is used to study hydrothermal characteristics of nano-fluid subjected to Brownian motion. For effective thermal conductivity and viscosity, Koo-Kleinstreuer-Li (KKL) model is used. It is observed that the dispersion of nano-particles in Newtonian liquid causes a significant increase in the effective thermal conductivity. The results based on the dispersion of nano-particles help engineers design an efficient thermal system. The significant role of viscous dissipation in diffusion of momentum of the wall into the fluid is observed. Therefore, effects of dissipations cannot be ignored while designing thermal systems. The buoyant force is responsible for the effect of electromagnetic thermal radiations on the fluid velocity. Convectively heated surface enhances the rate of generation of entropy. This study considers nano-fluids as the best coolants, compared to the base fluids. Imposition of magnetic field causes more entropy generation.
\end{abstract}

(C) 2019 Sharif University of Technology. All rights reserved.

\section{Introduction}

The efficiency enhancement of thermal systems has always been a major concern for technologists. Several techniques are used for this purpose. Besides conventional methods, some novel and efficient techniques for enhancing thermal efficiency are preferred for a number of reasons. One of the classical methods for enhancing heat transfer is to extend the surface of cooling fin; to do so, an increase in the size of cooling system, which is not desirable in many cases, is required. Therefore, engineers and scientists have introduced the technique of inclusion of nanosize metallic particles in the pure fluid. This causes the enhancement of thermal conductivity of the constituent fluid so that the thermal system can work in an efficient way. The inclusion of

\footnotetext{
*. Corresponding author.

E-mail address: Nawaz_d2006@yahoo.com (M. Nawaz)
}

nanosize metallic particles in a pure fluid gives rise to a mixture of fluid and totally different thermo-physical properties, compared to the pure fluid (the base fluid). This concept of dispersion of metallic nanosized particles has addressed many challenges of extension of surface of cooling fins. Invention of such fluids at the industrial level has motivated researchers to introduce imperial/mathematical models for the thermo-physical properties of nanofluids and, theoretically, studied the effects of nano-size metallic particles on the thermal conductivity enhancement of the resulting fluids. Such improvements in the process of heat transfer may cause a great revolution in thermal and cooling systems.

\subsection{Comparative analysis of models for effective diffusion coefficients}

In order to analyze the effects of dispersion of nano-size metallic particles on the effectiveness of viscosity and thermal conductivity of the pure fluid, different models have been used. Every model has some limitations. However, compared to the thermal performance of 
others, some models lead to lower thermal performance. These models include Einstein model [1], Brink model [2], Batchelor model [3], Mori and Ototake model [4], Wang et al. [5] model, Avsec and Oblak model [6], Masoumi et al. [7] model, etc. These models forecast the effectiveness of viscosity of a mixture of nanoparticles and fluid. These models provide correlations between viscosities (viscosity of base fluid, viscosity of nano-particles, and viscosity of nanofluid) and disregard the effective thermal conductivity of the mixture of nano-particles and base fluid. The use of effective viscosity models without considering theoretical models for effective thermal conductivity produces erroneous results. For this reason, theoretical models for effective viscosity and thermal conductivity are used simultaneously. The theoretical correlations for effective thermal conductivity can be found in [8-15]. However, these models predict that effective viscosity is a function of volume fraction. The role of Brownian motion of nano-particles in base fluid puts a bar on the above-mentioned models that do not exhibit the effect of Brownian motion of nano-particles on effective viscosity. This has motivated researchers to propose new models that are capable of exhibiting the effects of Brownian motion. Some new correlations considering the effects of Brownian motion and effectiveness of viscosity are proposed/used in [16-24].

However, the theoretical models used in [8-15] do not consider Brownian motion. Theoretical models for effective viscosity and thermal conductivity, as well as Brownian motion effect, are discussed in [1624]. However, the latest model is presented by Koo, Kleinstreuer and $\mathrm{Li}$ (KKL) [25] and is given by:

$$
\begin{aligned}
\rho_{n f} & =(1-\varphi) \rho_{f}+\varphi \rho_{s},\left(\rho c_{p}\right)_{n f} \\
& =(1-\varphi)\left(\rho c_{p}\right)_{f}+\varphi\left(\rho c_{p}\right)_{s} \\
\sigma_{n f} & =\sigma_{f}\left(1+\frac{3(\sigma-1) \varphi}{\sigma+2-(\sigma-1) \varphi}\right), \sigma=\frac{\sigma_{s}}{\sigma_{f}},
\end{aligned}
$$

Correlations for $k_{e f f}$ and $\mu_{n f}$ in the presence of Brownian motion are given as follows:

$$
\frac{k_{\text {static }}}{k_{f}}=1+\frac{3 \varphi\left(\frac{k_{p}}{k_{f}}-1\right)}{\left(\frac{k_{p}}{k_{f}}+2\right)-\left(\frac{k_{p}}{k_{f}}-1\right) \varphi},
$$

$$
\begin{aligned}
& k_{\text {eff }}=k_{\text {static }}+k_{\text {Brownian }}, \\
& k_{e f f}=1+\frac{3 \varphi\left(\frac{k_{p}}{k_{f}}-1\right)}{\left(\frac{k_{p}}{k_{f}}+2\right)-\left(\frac{k_{p}}{k_{f}}-1\right) \varphi}+5 \\
& \times 10^{4} g^{\prime}\left(\varphi, T, d_{p}\right) v \rho_{f}\left(c_{p}\right)_{f} \sqrt{\frac{\kappa_{b} T}{d_{p} \rho_{p}}} \\
& R_{f}=4 \times 10^{-8} \mathrm{~km}^{2} / W \\
& R_{f}=-d_{p}\left(1 / k_{p}-1 / k_{p, e f f}\right), \\
& g^{\prime}\left(\varphi, T, d_{p}\right)= \\
& \operatorname{Ln}(T)\left(\begin{array}{c}
a_{1}+a_{3} \operatorname{Ln}(\varphi)+a_{2} \operatorname{Ln}\left(d_{p}\right)+a_{5} \operatorname{Ln}\left(d_{p}\right)^{2} \\
+a_{4} \operatorname{Ln}\left(d_{p}\right) \operatorname{Ln}(\varphi)
\end{array}\right) \\
& +\left(\begin{array}{c}
a_{6}+a_{8} \operatorname{Ln}(\varphi)+a_{7} \operatorname{Ln}\left(d_{p}\right)+a_{10} \operatorname{Ln}\left(d_{p}\right)^{2} \\
+a_{9} \operatorname{Ln}(\varphi) \operatorname{Ln}\left(d_{p}\right)
\end{array}\right), \\
& k_{\text {Brownian }}=5 \times 10^{4} g^{\prime}\left(\varphi, T, d_{p}\right) \varphi \rho_{f}\left(c_{p}\right)_{f} \sqrt{\frac{\kappa_{b} T}{d_{p} \rho_{p}}},
\end{aligned}
$$$$
300 K<T<325 K
$$$$
\mu_{n f}=\frac{\mu_{f}}{(1-\varphi)^{2.5}}+\frac{k_{\text {Brownian }}}{k_{f}} \times \frac{\mu_{f}}{\operatorname{Pr}_{f}} .
$$

Thermo-physical properties of water and two types of metallic nano-particles, which are used by $\mathrm{Li}$ and Sheikholeslami [26], are given in Table 1, and coefficient values of copper oxide and aluminum oxide nanofluids are shown in Table 2.

This model has been used in some recent studies. For example, Sheikholeslami et al. [26] studied heat transfer enhancement in turbulent flow caused by twisted-tape turbulators using the KKL model. Sheikholeslami [27] analyzed the effect of nano-particles and Brownian motion on the transfer of heat in a square closure. In this study, Sheikholeslami [27] augmented nanofluid characteristics by using the KKL model for effective viscosity and thermal conductivity. Another study was conducted by Sheikholeslami [28] in order to explore the process of solidification in nanofluid

Table 1. Thermo-physical properties of water and nanoparticles used in [25,28].

\begin{tabular}{lccc}
\hline Physical property & Water/base fluid & $\mathbf{C u O}$ & $\mathbf{A l}_{\mathbf{2}} \mathbf{O}_{\mathbf{3}}$ \\
\hline$\rho\left(\mathrm{kg} \cdot \mathrm{m}^{-3}\right)$ & 997.1 & 6500 & 3970 \\
$c_{p}\left(\mathrm{~J} \cdot \mathrm{kg}^{-1} \cdot \mathrm{K}^{-1}\right)$ & 4179 & 540 & 765 \\
$k\left(\mathrm{~W} \cdot \mathrm{m}^{-1} \cdot \mathrm{K}^{-1}\right)$ & 0.613 & 18 & 25 \\
$d_{p}(\mathrm{~nm})$ & - & 47 & 29 \\
$\sigma\left(\mathrm{s} \cdot \mathrm{m}^{-1}\right)$ & $5.5 \times 10^{-6}$ & $1 \times 10^{-10}$ & $35 \times 10^{6}$ \\
\hline
\end{tabular}


Table 2. The values of coefficients for $\mathrm{CuO}$ and $\mathrm{Al}_{2} \mathrm{O}_{3}$ nanofluids [25,28].

\begin{tabular}{cll}
\hline Coefficient values & \multicolumn{1}{c}{$\mathbf{C u O}$-water } & \multicolumn{1}{c}{$\mathbf{A l}_{\mathbf{2}} \mathbf{O}_{\mathbf{3}}$-water } \\
\hline$a_{1}$ & -26.593310846 & 52.813488759 \\
$a_{2}$ & -0.403818333 & 6.115637295 \\
$a_{3}$ & -33.3516805 & 0.6955745084 \\
$a_{4}$ & -1.915825591 & $4.17455552786 \times 10^{-2}$ \\
$a_{5}$ & $6.42185846658 \times 10^{-2}$ & 0.176919300241 \\
$a_{6}$ & 48.40336955 & -298.19819084 \\
$a_{7}$ & -9.787756683 & -34.532716906 \\
$a_{8}$ & 190.245610009 & -3.9225289283 \\
$a_{9}$ & 10.9285386565 & -0.2354329626 \\
$a_{10}$ & -0.72009983664 & -0.999063481 \\
\hline
\end{tabular}

by considering the KKL method. Sheikholeslami and Rokni [29] used Control Volume Finite Element Approach (CVFEA) to investigate the effect of Lorentz force on the flow of nanofluid in a porous enclosure using a non-equilibrium model together with the KKL model for effective viscosity and thermal conductivity. Sheikholeslami et al. [30] studied the effects of Darcy porous medium on the MHD flow in a complex-shaped container containing $\mathrm{Al}_{2} \mathrm{O}_{3}$-nanofluid while estimating effective viscosity and thermal conductivity through the KKL model. Sheikholeslami with other contributors used the KKL model for effective viscosity and thermal conductivity. These studies are referenced in [31-38]. Some latest studies on nanofluid flows are mentioned in [39-57].

Minimization of the entropy generation in the thermal system is a major concern as a waste of energy causes a great disorder. Therefore, the control of entropy generation during heat transfer has been investigated extensively over the last few years. Bejan [58] was the first to work on the minimization of entropy generation. After this pioneering work, several studies have been published. However, here, some recent investigations are described as follows. For instance, Bhatti et al. [59] investigated the effects of magnetic field on the entropy generation of nonlinear transport of heat and mass in the boundary layer flow. Numerical investigation of entropy generation during the heat transfer in the cavity flow was carried out by Armaghani et al. [60]. Bianco et al. [61] analyzed the effect of entropy generation due to temperature difference and viscosity/friction losses in the flow.

Literature review reveals that no FEM study on hydro-thermal characteristics in nano-fluid subjected to Lorentz force, thermal radiation, buoyancy force using the KKL model has been investigated so far. A comprehensive literature review is given in Section 1. Modeling of unsteady flow of nanofluid in the presence of buoyancy force and Joule heating is given in
Section 2. Galerkin weak formulation and coefficients of stiffness matrix are given in Section 3. Section four is associated with results and discussion. Entropy analysis is also given in Section 4. Key points of this study are listed in Section 5.

\section{Mathematical models and modeling}

\subsection{Problem statement}

Let us investigate the effects of dispersion of nanoparticles $\left(\mathrm{CuO}\right.$ and $\mathrm{Al}_{2} \mathrm{O}_{3}$ ) on the performance of thermal conductivity and viscosity using the KKL model. The flow over the sheet results from the unsteady motion of the sheet moving with velocity $U_{w}(x, t)=a x /(1-c t)$ and is subjected to the magnetic field. Nanofluid is assumed to exhibit thermal radiation and generates heat during thermal changes. Buoyant force is a considerable order of magnitude. Thermophysical properties (viscosity, density, thermal conductivity, specific heat, etc.) are constant. The transport of heat nanofluid (occupying half space $y>0$ ) is due to convection from the hot fluid (occupying half space $y<0)$ of temperature $T_{w}(x, t)=T_{\infty}+a x /(1-c t)$. The buoyant force under Boussinesq approximation is significant, as shown in Figure 1.

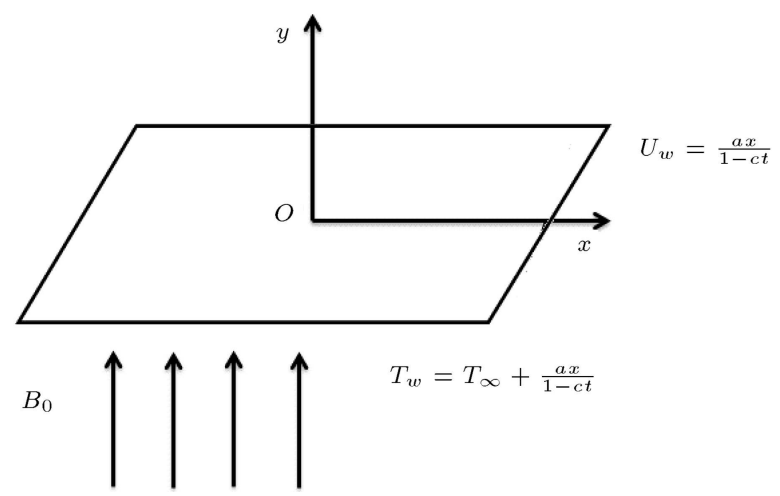

Figure 1. Physical model and coordinates system. 


\subsection{Diffusion models}

Mass, momentum, and thermal diffusion models under boundary layer approximations are as follows [51,52]:

$$
\begin{aligned}
& \frac{\partial u}{\partial x}+\frac{\partial v}{\partial y}=0, \\
& \frac{\partial u}{\partial t}+u \frac{\partial u}{\partial x}+v \frac{\partial u}{\partial y}=\frac{1}{\rho_{n f}}\left(\mu_{n f} \frac{\partial^{2} u}{\partial^{2} y}\right)-\frac{\sigma_{n f} B_{0}^{2} u}{\rho_{n f}} \\
& \quad+\beta_{T} g\left(T-T_{\infty}\right), \\
& \frac{\partial T}{\partial t}+u \frac{\partial T}{\partial x}+v \frac{\partial T}{\partial y}=\frac{1}{\left(\rho c_{p}\right)_{n f}}\left[k_{e f f} \frac{\partial^{2} T}{\partial^{2} y}\right. \\
& \left.\quad+Q\left(T-T_{\infty}\right)\right]+\frac{\sigma_{n f} B_{0}^{2}}{\left(\rho c_{p}\right)_{n f}} u^{2}-\nabla \cdot \overrightarrow{\mathbf{q}} .
\end{aligned}
$$

Stefan Boltzmann law is defined by:

$$
\overrightarrow{\mathbf{q}}=-\frac{4 \sigma^{*}}{3 k^{*}} \nabla\left(T^{4}-T_{\infty}^{4}\right) \text {. }
$$

The initial and boundary conditions are obtained by Eqs. (6) and (7) as shown in Box I.

\subsection{Normalization of equations}

Diffusion Eqs. (3)-(5) and initial, boundary conditions (6) and (7) are made dimensionless by applying the following transformations:

$$
\left.\begin{array}{c}
u=\frac{\partial \psi}{\partial y}, v=-\frac{\partial \psi}{\partial x}, \psi(x, y)=\sqrt{\left(\frac{a \nu_{f}}{(1-c t)}\right)} x f(\eta), \\
\theta(\eta)=\frac{T-T_{\infty}}{T_{w}-T_{\infty}}, \eta=\sqrt{\left(\frac{a}{v_{f}(1-c t)}\right)} y,
\end{array}\right)
$$

Hence, we get:

$$
\begin{gathered}
f^{\prime \prime \prime}-\frac{A_{5}}{A_{3}} M^{2} f^{\prime}+\frac{A_{1}}{A_{3}} \operatorname{Gr} \theta-\frac{A_{1}}{A_{3}} \\
{\left[f^{\prime 2}-f f^{\prime \prime}+\lambda\left(f^{\prime}+\frac{1}{2} \eta f^{\prime \prime}\right)\right]=0,} \\
f(0)=0, f^{\prime}(0)=1, f^{\prime} \rightarrow 0, \\
\left(1+\frac{4}{3 N_{r}}\right) \theta^{\prime \prime}+\operatorname{Pr} \frac{1}{A_{4}} \beta \theta+\frac{A_{3} A_{5}}{A_{4}} M^{2} \operatorname{Pr} \operatorname{Ec} f^{\prime 2} \\
\quad+\frac{A_{2}}{A_{4}} \operatorname{Pr}\left[f \theta^{\prime}-f^{\prime} \theta-\lambda\left(2 \theta+\frac{1}{2} \eta \theta\right)\right]=0,
\end{gathered}
$$

$$
\theta^{\prime}(0)=-B_{i}(1-\theta(0)), \quad \theta \rightarrow 0 \quad \text { as } \quad \eta \rightarrow \infty
$$

where:

$$
\begin{array}{ll}
A_{1}=\frac{\rho_{n f}}{\rho_{f}}, & A_{2}=\frac{\left(\rho c_{p}\right)_{n f}}{\left(\rho c_{p}\right)_{f}}, \quad A_{3}=\frac{\mu_{n f}}{\mu_{f}}, \\
A_{4}=\frac{k_{e f f}}{k_{f}}, & A_{5}=\frac{\sigma_{n f}}{\sigma_{f}}
\end{array}
$$

and:

$$
\begin{aligned}
& M^{2}=\frac{\sigma_{f} B_{o}^{2}(1-c t)}{a \rho_{f}}, \\
& \mathrm{Gr}=\frac{\beta_{T}\left(T_{w}-T_{\infty}\right)(1-c t)^{2}}{a^{2} x}, \\
& \lambda=\frac{c}{a}, \quad N_{r}=\frac{k_{e f f} k^{*}}{4 \sigma^{*} T_{\infty}^{3}}, \\
& \operatorname{Pr}=\frac{\mu_{f}\left(c_{p}\right)_{f}}{k_{f}}, \quad \operatorname{Ec}=\frac{\left(\frac{a x}{1-c t}\right)^{2}}{\left(c_{p}\right)_{f}\left(T_{w}-T_{\infty}\right)}, \\
& B_{i}=\frac{h_{f}}{k_{f}} \sqrt{\frac{(1-c t) v_{f}}{a}}, \quad \beta=\frac{Q_{0}}{a\left(\rho c_{p}\right)_{f}},
\end{aligned}
$$

are the Hartmann number, the Grashof number, the unsteadiness parameter, the radiation parameter, the Prandtl number, the Eckert number, the Biot number, and heat generation/absorption parameter, respectively. The prescribed wall temperature case can be recovered as $B_{i} \rightarrow \infty$. Further, note that $k_{\text {Brownian }}=$ 0 and $\varphi=0$ is the case when fluid is pure and nanoparticles are not dispersed (the case of Butt and Ali, $62]$ ), and for $\mathrm{Gr}=0, N_{r}=0$ and $\mathrm{Ec}=0$, the problem is reduced to the case of Das et al. [63] with heat generation/absorption. The case of $M^{2}=0, \lambda=0$, $k_{\text {Brownian }}=0, \varphi=0$ and $B_{i} \rightarrow \infty$ is also considered by Abolbashari et al. [64] and Das et al. [63].

\section{Numerical method}

Galerkin Finite Element Method (GFEM) is implemented to carry out simulations of heat transfer through dimensionless conservation. As a part of the procedure, the following steps will be taken.

$$
\begin{gathered}
\begin{array}{l}
u=v=0, \quad T=T_{\infty}, \quad \forall x, y, t<0 \\
\left.\begin{array}{c}
u(x, y)=U_{w}(x, t), v=0,-k_{f} \frac{\partial T}{\partial y}=h_{f}\left[T_{w}(x, t)-T\right], \text { at } \quad y=0, \\
u \rightarrow 0, T \rightarrow T_{\infty} \quad \text { as } y \rightarrow \infty .
\end{array}\right\} t>0 .
\end{array}
\end{gathered}
$$




\subsection{Domain discretization}

The physical domain (after dimensional analysis) $[0, \infty]$ is divided into line elements with two nodes per elements.

\subsection{Selection of weight and interpolation functions}

As there are two nodes per element, weight and shape functions (interpolation functions) are selected in the linear form. Further, as suggested by the Galerkin approach, weight functions are taken equal to the interpolation functions. The following linear interpolation functions are defined as follows:

$$
\psi_{j}=(-1)^{j-1}\left(\frac{\eta_{j+1}-\eta}{\eta_{j+1}-\eta_{j}}\right), j=1,2 .
$$

\subsection{Constructions of residual equations}

Residual equations are defined and multiplied by weights. The resulting weighted residuals are integrated over a typical element $\left[\eta_{e}, \eta_{e+1}\right]$.

\subsection{Weak form of weighted residuals}

The weighted integral residuals are integrated over the line element to convert the strong form of weighted residual into the weak form.

\subsection{Derivation of stiffness coefficients}

The dependent unknowns are approximated over element $\left[\eta_{e}, \eta_{e+1}\right]$ by the finite element approximations. The unknown nodal values are computed. Considering certain approximations used in weak formulation of weighted residuals, one can obtain the finite element model of the form:

$$
\left[K^{e}\{\pi\}\right]\left[\pi^{e}\right]=\left\{F^{e}\right\}+\left\{Q^{e}\right\},
$$

where $\left[K^{e}\{\pi\}\right]$ is the stiffness matrix for typical element, $\pi^{e}$ represents the unknown nodal values, $\left\{F^{e}\right\}$ is the boundary vector, and $\left\{Q^{e}\right\}$ is the source vector.

\subsection{Assembly process}

Following the assembly procedure of finite element approach, the system of nonlinear algebraic equations of the form is obtained as follows:

$$
[K\{\pi\}]\{\pi\}=\{F\}
$$

where $[K\{\pi\}]$ is the global coefficient matrix. It is important to note that the coefficients of stiffness matrix $[K\{\pi\}]$ are also functions of unknown nodal values. Therefore, systems of algebraic equations (12) are solved numerically by an iterative procedure. Here, in this study, Picard's linearization procedure is used, which works in the following way:

$$
\left[K\{\pi\}^{r-1}\right]\{\pi\}^{r}=\{F\}
$$

where $\{\pi\}^{r-1}$ represents the nodal values computed at the $(r-1)$ th iteration, and $\{\pi\}^{r}$ represents the nodal values computed at the $r$ th iteration.

\subsection{Computer implementation}

The linearized system of algebraic equations (15) is solved iteratively by a Guass-Siedal approach. The computational procedure described above is implemented using Matlab. The developed computer code works with tolerance $10^{-5}$; several computational experiments were carried out to search the value of $\eta$, where asymptotic boundary conditions are satisfied. Simulations carried out in this study reveal that the asymptotic boundary conditions are satisfied when $\eta=$ 6. Therefore, $\eta_{\max }=6$ is taken as infinity, i.e., the computational domain is $[0,6]$.

\subsection{Convergence and error analysis}

The following error in the simulated results is calculated as follows:

$$
\text { error }=\left|\pi^{r}-\pi^{r-1}\right|
$$

and convergence criteria are set in the following form:

$$
\max \left|\pi_{i}^{r}-\pi_{i}^{r-1}\right|<\xi
$$

where $\xi$ is the tolerance and is taken equal to $10^{-5}$ in this coming analysis.

\subsection{Validation}

To validate the results, the numerical values of $-\theta^{\prime}(0)$ for special case (for different $\operatorname{Pr}$ when $M^{2}=\lambda=\varphi=$ $k_{\text {Brwonian }}=0$, and $B_{i} \rightarrow \infty$ ) are compared with the already published benchmarks by Das et al. [63] and Albashari et al. [64]. This comparison is displayed in Table 3. This table guarantees excellent agreement between the present and already published works.

Table 3. Validation of the present results by comparing them with those published by Albashari et al. [64] and Das et al. [63] of $-\theta^{\prime}(0)$ for different $\operatorname{Pr}$ when $M^{2}=\lambda=\varphi=k_{\text {Brwonian }}=0$, and $B_{i} \rightarrow \infty$.

\begin{tabular}{cccc}
\hline Pr & Abolbashari et al. [64] & Das et al. [63] & Present study \\
\hline 0.72 & 0.80863135 & 0.80876122 & 0.74454088 \\
1.00 & 1.00000000 & 1.00000000 & 0.91192959 \\
3.00 & 1.92368259 & 1.92357431 & 1.81548127 \\
7.00 & 3.07225021 & 3.07314679 & 2.96744744 \\
10.0 & 3.72067390 & 3.72055436 & 3.61534147 \\
\hline
\end{tabular}




\section{Results and discussion}

In order to analyze the physics of the described flow situation, the computed field variables versus the physical parameters are displayed. Figure 2 displays the effect of magnetic field on the flow of $\mathrm{CuO}$ nanoliquid (solid curves) and $\mathrm{Al}_{2} \mathrm{O}_{3}$ nanoliquid (dotted curves). Hartmann number is the ratio of Lorentz force to inertial force, and an increase in Hartmann number corresponds to an increase in magnetic field intensity. Hence, it is observed that flow experiences move retardation when magnetic field intensity increases. It is also noted that $\mathrm{Al}_{2} \mathrm{O}_{3}$ nanoliquid experiences more Lorentz force than the $\mathrm{CuO}$ nanoliquid (see Figure 2). Behavior of the flow of $\mathrm{Al}_{2} \mathrm{O}_{3}$ and $\mathrm{CuO}$ nanoliquids under an increase in the buoyant force is represented by Figure 3. During simulations, it is also observed

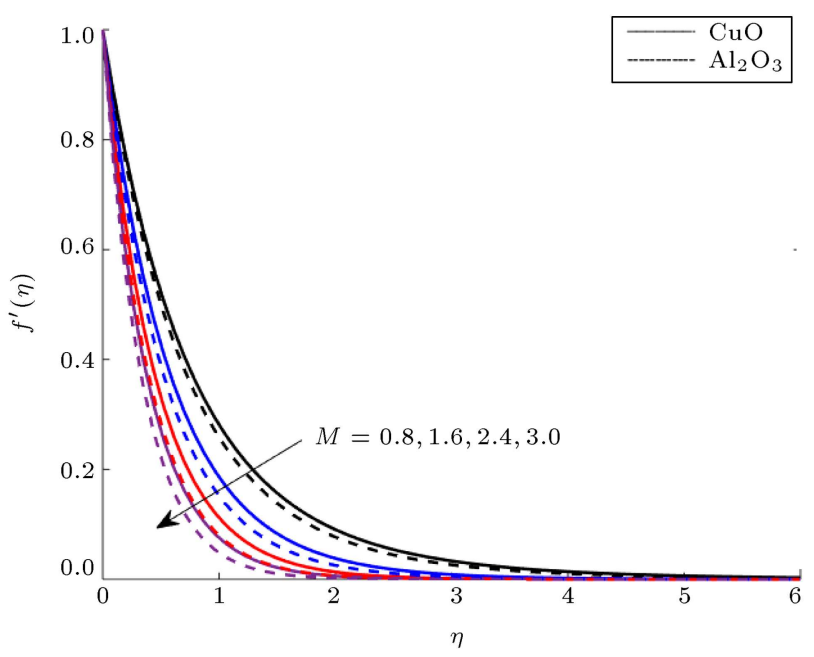

Figure 2. Flow behavior versus $M$ when $\mathrm{Gr}=4, \operatorname{Pr}=2.73, \mathrm{Ec}=0.1, N_{r}=0.2, B_{i}=0.1$, $\varphi=0.04, \beta=0.9$, and $\lambda=0.6$.

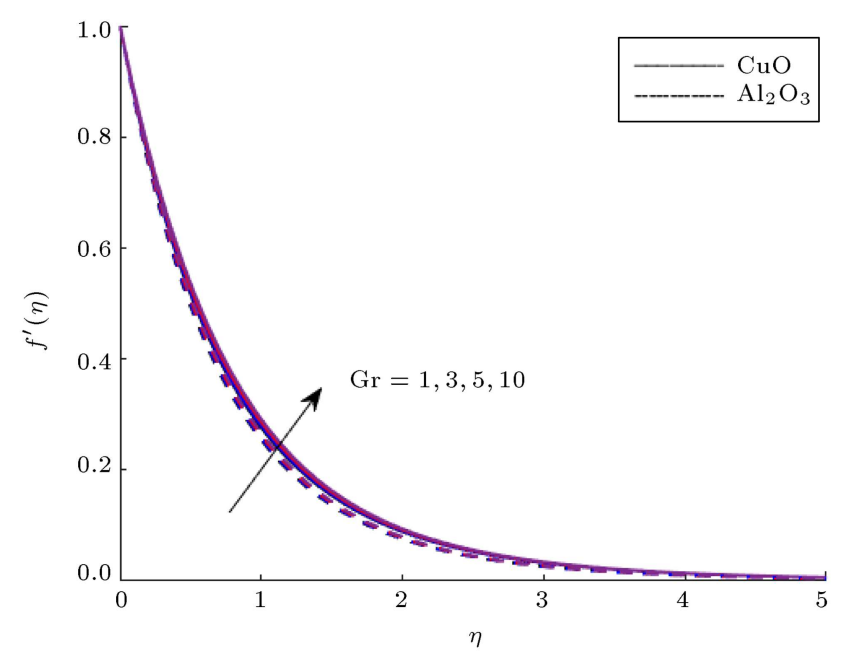

Figure 3. Flow behavior versus $\mathrm{Gr}$ when $\operatorname{Pr}=2.73, \mathrm{Ec}=0.1, N_{r}=0.2, M=0.8, B_{i}=0.1$, $N_{r}=0.2, \varphi=0.04, \beta_{1}=0.9$, and $\lambda=0.6$. that flow is assisted by positive buoyant force $(\mathrm{Gr}>0)$, whereas it is opposed by negative buoyant force $(\mathrm{Gr}<$ $0)$. The impact of positive buoyant force on $\mathrm{Al}_{2} \mathrm{O}_{3}$ nanoliquid flow is more than that of buoyant force on nanoliquid flow, as shown in Figure 3. The velocity field is subject to a decreasing trend by increasing the unsteadiness parameter, $\lambda$, as represented in Figure 4. The velocity of steady flow is greater than that of unsteady flow. The boundary layer thickness decreases the function of the unsteadiness parameter, $\lambda$. The intensity and heat dissipations due to Joule heating are directly proportional. Therefore, an increase in the intensity of magnetic field enhances the dissipation rate of heat in the liquid regime and, thus, temperature increases. The effect of intensity of magnetic field on the temperature is displayed in Figure 5 for both

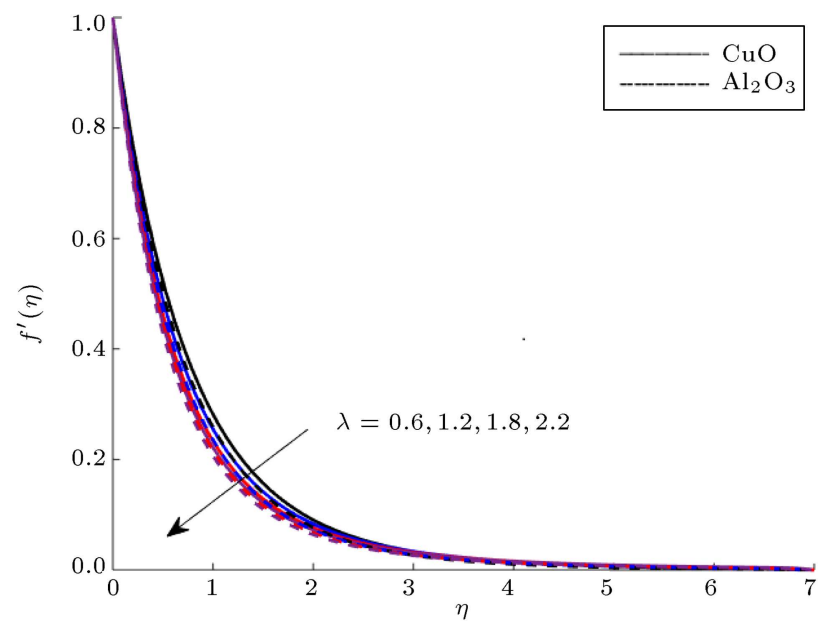

Figure 4. Unsteadiness behavior for fluid when $\operatorname{Pr}=2.73, \mathrm{Ec}=0.1, N_{r}=0.2, M=0.8, B_{i}=0.1$, $N_{r}=0.2, \varphi=0.04, \beta=0.9$, and $\mathrm{Gr}=4$.

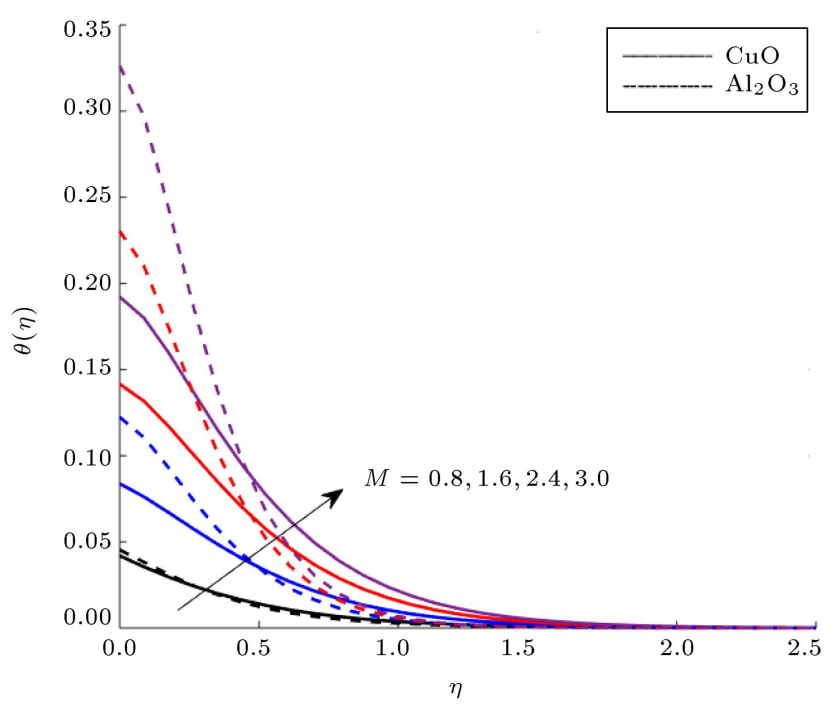

Figure 5. Temperature curves versus $M$ when $\mathrm{Gr}=4, \operatorname{Pr}=2.73, \mathrm{Ec}=0.1, N_{r}=0.2, \varphi=0.04$, $B_{i}=0.1, \beta=0.9$, and $\lambda=0.6$. 
$\mathrm{Al}_{2} \mathrm{O}_{3}$ nanoliquid and $\mathrm{CuO}$ nanoliquid. According to Figure 5, it can also be observed that the dissipation rate of heat to Ohmic heating (heating due to magnetic field) in $\mathrm{Al}_{2} \mathrm{O}_{3}$ nanoliquid regime is greater than that in $\mathrm{CuO}$ nanoliquid when $\mathrm{Gr}=4, \mathrm{Pr}=2.73, \mathrm{Ec}=$ $0.1, N_{r}=0.2, \varphi=0.04, B_{i}=0.1, \beta=0.9$, and $\lambda=0.6$. The Eckert number, Ec, appears as a coefficient of Joule heating term in the dimensionless form of energy equation (see Eq. (10)), and an increase in it causes the enhancement rate of Joule heating. Consequently, the temperature increases. This fact is displayed in Figure 6. The comparison of Figures 5 and 6 shows that the temperature of fluids varies in a similar fashion, in a qualitative sense, when Hartmann and $E c$ are increased. The temperature field for both $\mathrm{Al}_{2} \mathrm{O}_{3}$ nanoliquid (dotted curves) and $\mathrm{CuO}$ nanoliquid (solid curves) under a variation of unsteadiness parameter, $\lambda$, is reflected by Figure 7 . This figure shows the declining behavior of temperature with respect to the unsteadiness parameter, $\lambda$. Figure 8 displays the temperature curves for $\mathrm{CuO}$ and $\mathrm{Al}_{2} \mathrm{O}_{3}$. These temperature curves show that $\mathrm{Al}_{2} \mathrm{O}_{3}$ nanoparticles are responsible for more heat transfer than nanoparticles. The temperature distribution for both $\mathrm{CuO}$ nanoliquid and $\mathrm{Al}_{2} \mathrm{O}_{3}$ nanoliquid due to heat generation in the liquid regime is given in Figure 9. A significant increase in temperature due to internal heat generation is observed. The role of convective boundary in enhancing transfer of heat from heated wall to the nanoliquid regime is revealed in Figure 10. Accordingly, the heat transfer process speeds up if the convection parameter (Biot number) is increased. However, this convection is more significant in $\mathrm{CuO}$ nanoliquid than that in $\mathrm{Al}_{2} \mathrm{O}_{3}$ nanoliquid. Thermal radiation effects of the temperature of $\mathrm{Al}_{2} \mathrm{O}_{3}$ nanoliquid and nanoliquid are shown in Figure 11. It can be easily noted that the heat

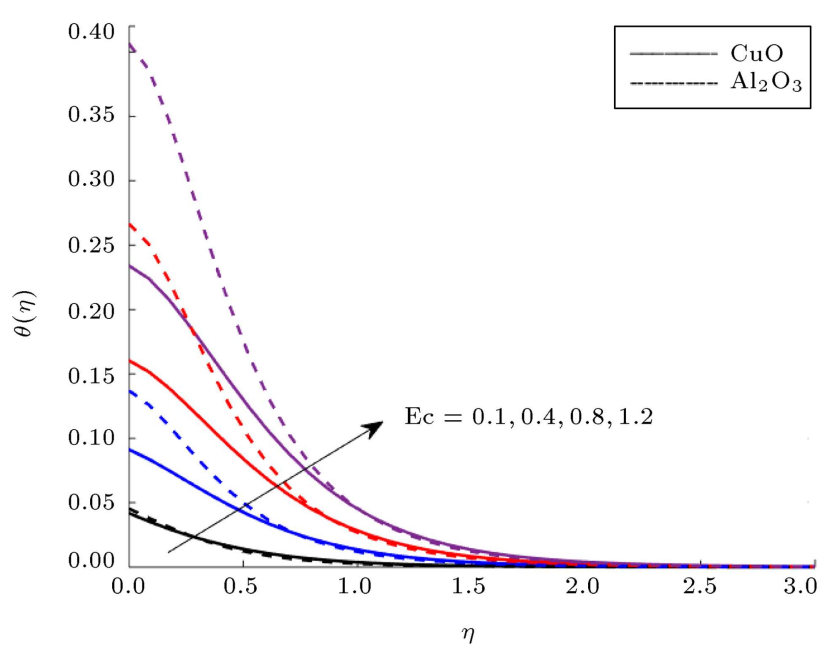

Figure 6. Temperature curves versus Ec when $\mathrm{Gr}=4, \mathrm{Pr}=2.73, N_{r}=0.2, B_{i}=0.1, M=0.8$, $N_{r}=0.2, \varphi=0.04, \beta=0.9$, and $\lambda=0.6$.

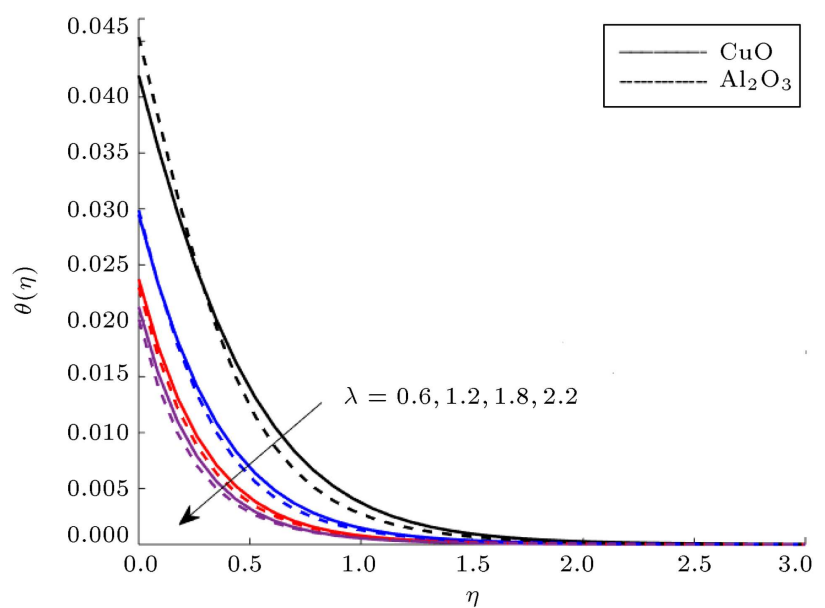

Figure 7. Temperature curves versus $\lambda$ when $\mathrm{Gr}=4, \beta=0.9, \operatorname{Pr}=2.73, \mathrm{Ec}=0.1, N_{r}=0.2$, $\varphi=0.04, M=0.8$, and $B_{i}=0.1$.

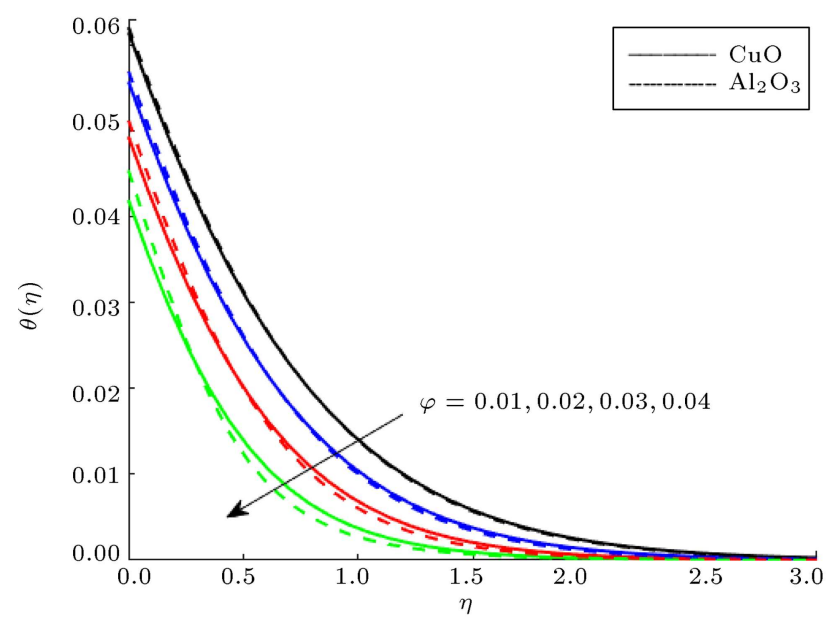

Figure 8. Temperature curves versus $\varphi$ when $\mathrm{Gr}=4, \operatorname{Pr}=2.73, \mathrm{Ec}=0.1, N_{r}=0.2, B_{i}=0.1$, $M=0.8, \beta=0.9$, and $\lambda=0.6$.

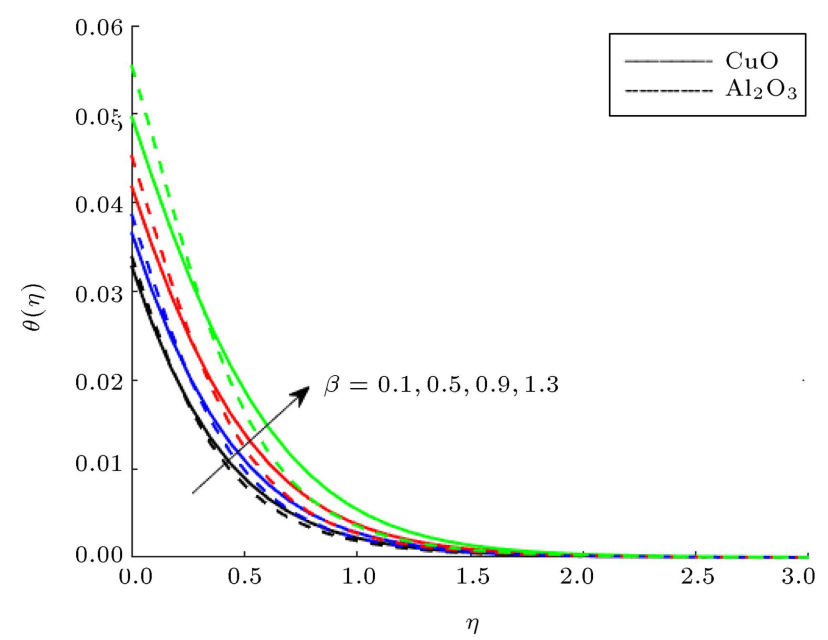

Figure 9. Temperature curves versus $\beta$ when $\mathrm{Gr}=4, \operatorname{Pr}=2.73, \mathrm{Ec}=0.1, N_{r}=0.2, B_{i}=0.1$, $\varphi=0.04, M=0.8$, and $\lambda=0.6$. 


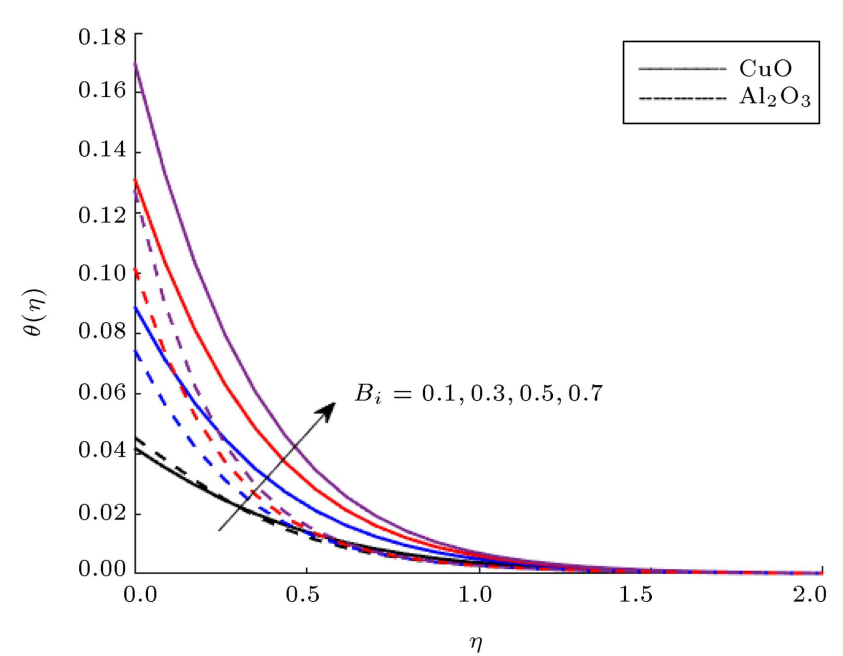

Figure 10. Temperature curves versus $B_{i}$ when $\mathrm{Gr}=4, \operatorname{Pr}=2.73, \mathrm{Ec}=0.1, N_{r}=0.2, \varphi=0.04, M=0.8$, and $\lambda=0.6$.

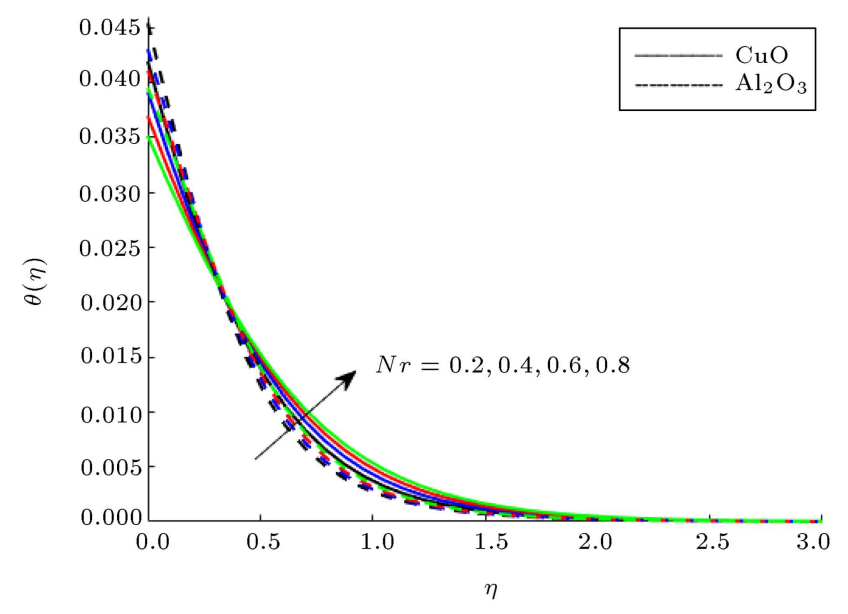

Figure 11. Temperature curves versus $N_{r}$ when $\mathrm{Gr}=4, \operatorname{Pr}=2.73, \mathrm{Ec}=0.1, B_{i}=0.1, \beta=0.9$, $\varphi=0.04, M=0.8$, and $\lambda=0.6$.

transfer process increases if the radiation parameter $\left(N_{r}\right)$ increases.

\section{Entropy generation}

\subsection{Entropy analysis}

The entropy generation due to temperature gradient, viscous dissipation, and Joule heating is defined by

$$
\begin{aligned}
E_{G}= & \frac{k_{e f f}}{T_{\infty}^{2}}\left[\left(\frac{\partial T}{\partial x}\right)^{2}+\left(\frac{\partial T}{\partial y}\right)^{2}\right]+\frac{\mu_{n f}}{T_{\infty}}\left(\frac{\partial u}{\partial y}\right)^{2} \\
& +\frac{\sigma_{n f} B_{o}^{2}}{T_{\infty}} u^{2}
\end{aligned}
$$

Using the similarity transformations given in Eq. (8), one obtains the following dimensionless form of entropy generation.

$$
\begin{aligned}
N_{S}= & \operatorname{Re} \frac{k_{\text {eff }}}{k_{f}} \theta^{\prime^{2}}+\frac{\mathrm{Br}}{\left((1-\varphi)^{\frac{5}{2}}+\frac{k_{\text {Brownian }}}{k_{f}} \times \frac{1}{\operatorname{Pr}_{f}}\right) \Omega} \\
& {\left[f \prime^{2}+\varphi_{2} M^{2} f^{\prime 2}\right] }
\end{aligned}
$$

where:

$$
\begin{array}{ll}
N_{S}=\frac{T_{f}^{2} a^{2} E_{G}}{k_{f}\left(T_{w}-T_{\infty}\right)^{2}}, & \operatorname{Re}=\frac{U_{w} a}{v_{f} x}, \\
\mathrm{Br}=\frac{\mu_{f} U_{w}^{2}}{k_{f}\left(T_{w}-T_{\infty}\right)^{2}}, & \Omega=\frac{T_{w}-T_{\infty}}{T_{f}},
\end{array}
$$

are called dimensionless entropy generation number, the Reynolds number, the Brinkman number, and the non-dimensionless temperature difference number, respectively.

\subsection{Entropy generation profiles}

The effect of an increase in magnetic field intensity on the entropy generation is shown in Figure 12 . This graphical behavior of entropy generation versus Hartmann number reveals that external magnetic field enhances the entropy generation rate. It is predicted that the imposition of magnetic field to thermal system causes greater energy loss. Thus, it can be concluded that magnetic field should not be imposed if energy losses are to be minimized as is required for industrial processes. Based on Figure 12, entropy generation in $\mathrm{Al}_{2} \mathrm{O}_{3}$ nanoliquid is greater than that in $\mathrm{CuO}$ nanoliquid. An increasing trend in entropy generation is observed when temperature difference parameter is increased (see Figure 13). The entropy production is greatly influenced by an increase in the Reynolds number. An increasing trend in entropy generation due to an increase in Reynolds can be seen in Figure 14.

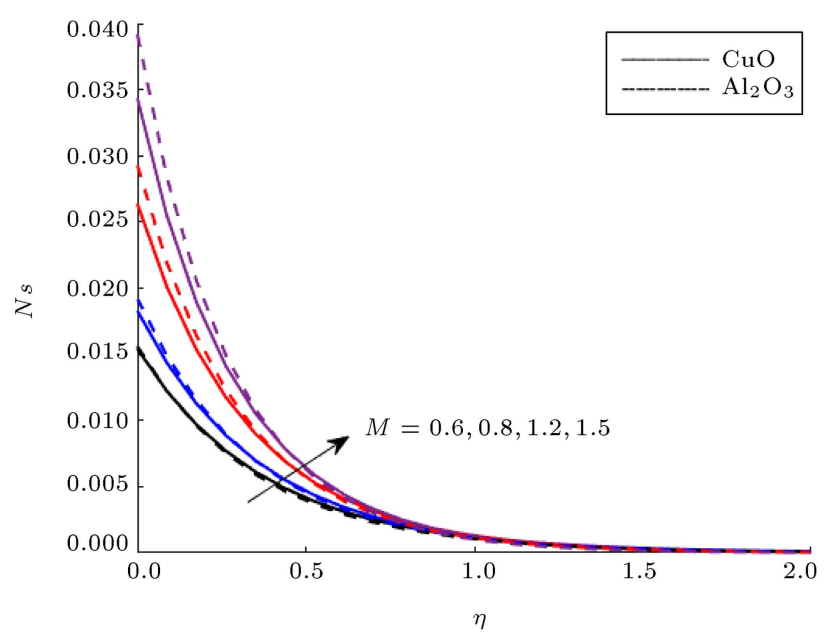

Figure 12. The effect of $M$ on the entropy generation when $\mathrm{Gr}=4, \operatorname{Pr}=2.73, \mathrm{Ec}=0.1, B_{i}=0.1$, $\beta=0.9, N_{r}=0.2, \lambda=0.9, \operatorname{Re}=1, \operatorname{Br}=1$, and $\Omega=1$. 


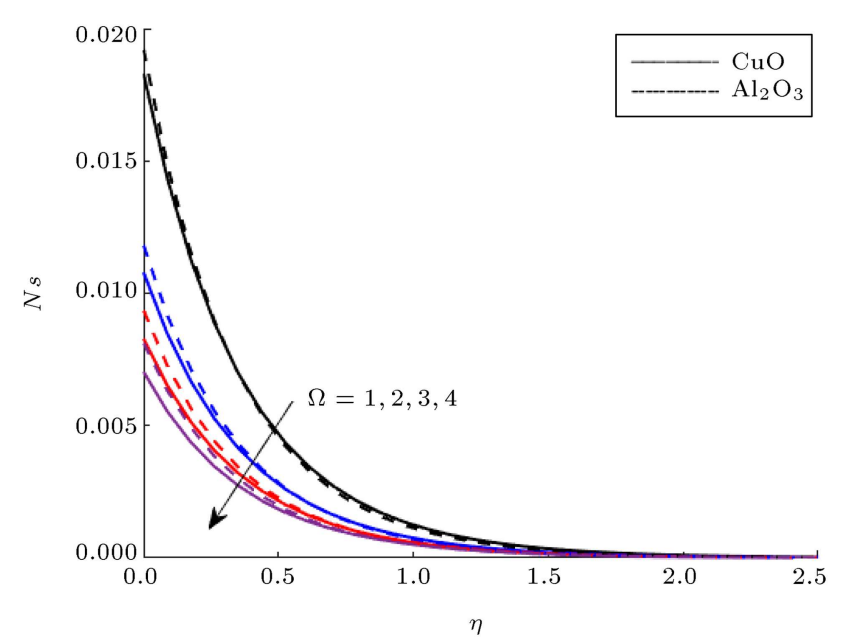

Figure 13. The effect of $\Omega$ on the entropy generation when $\mathrm{Gr}=4, \operatorname{Pr}=2.73, N_{r}=0.2, B_{i}=0.1$, $\beta=0.9, M=0.8, \lambda=0.9, \operatorname{Re}=1, \mathrm{Br}=1$, and $\mathrm{Ec}=0.1$.

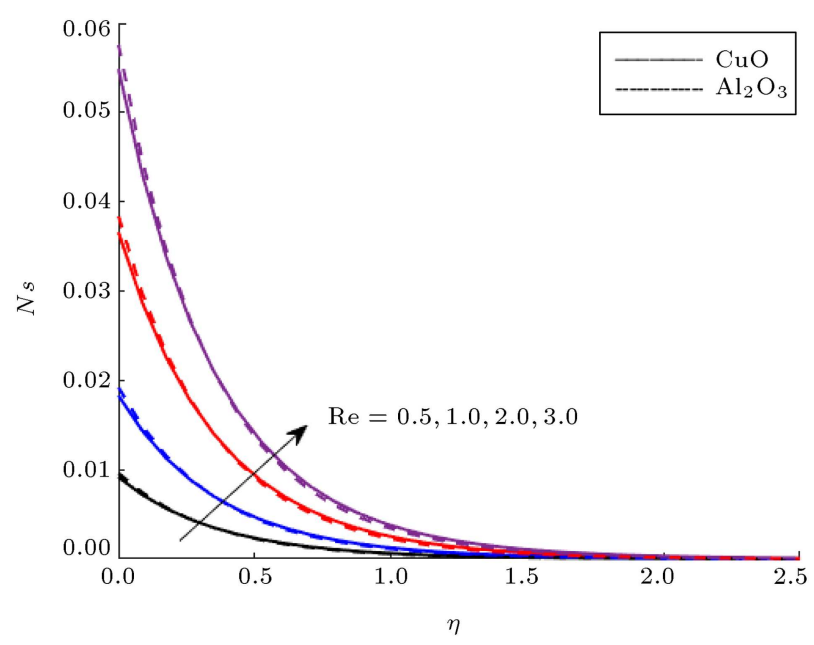

Figure 14. The effect of Re on the entropy generation when $\mathrm{Gr}=4, \mathrm{Pr}=2.73, \mathrm{Ec}=0.1, \beta=0.9, M=$ $0.8, N_{r}=0.2, \lambda=0.9, \mathrm{Br}=1$, and $\Omega=1$.

Figure 15 depicts that entropy generation increases when Ec increases.

\section{Conclusion}

The effects of effective viscosity and thermal conductivity on the enhancement rate of heat transfer due to the dispersion of nano-sized metallic particles $(\mathrm{CuO}$ and $\left.\mathrm{Al}_{2} \mathrm{O}_{3}\right)$ were studied by the KKL model for Brownian motion. A significant increase in thermal conductivity was observed. $\mathrm{Al}_{2} \mathrm{O}_{3}$ nanoliquid experienced a greater resistive force due to the magnetic field than $\mathrm{CuO}$ nanoliquid did. The effects of four types of nanoparticles $\left(\mathrm{Cu}, \mathrm{Ag}, \mathrm{Al}_{2} \mathrm{O}_{3}\right.$ and $\mathrm{TiO}_{2}$ ) on the transfer of heat in unsteady two-dimensional boundary layer flow of a radiative fluid on a convectively heated surface in the presence of Joule heating, heat absorp-

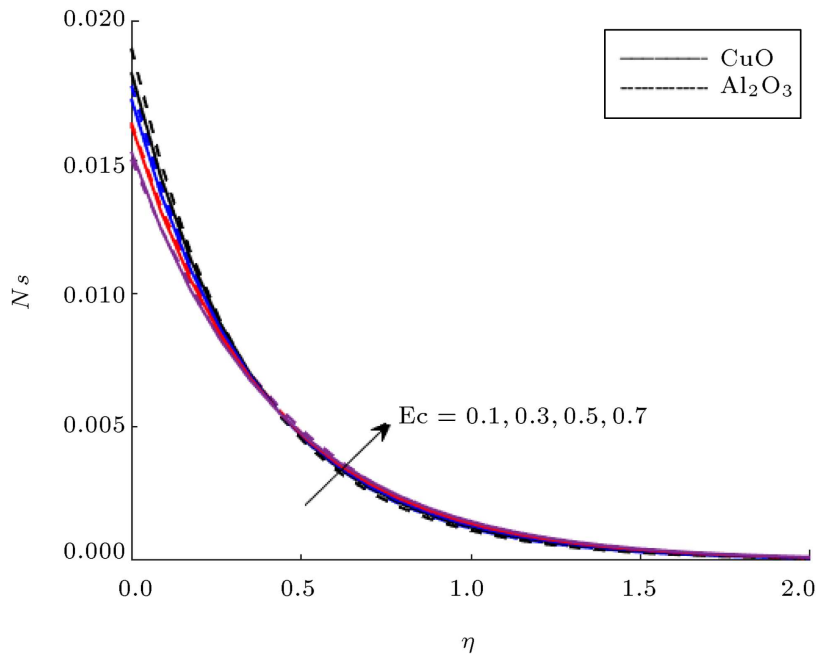

Figure 15. The effect of Ec on the entropy generation when $\mathrm{Gr}=4, \operatorname{Pr}=2.73, N_{r}=0.2, B_{i}=0.1$, $\beta=0.9, M=0.8, \lambda=0.9, \operatorname{Re}=1, \mathrm{Br}=1$, and $\omega=1$.

tion/generation, and buoyant force were investigated. It was observed that dispersion of nano-particles in the pure fluid increased the thermal conductivity of the resulting mixture, which may play a vital role in thermal systems. To ensure favorable buoyant force, the velocity of the mixture (mixture of nanoparticles and radiative fluid) increased, causing an increase in the thermal and momentum boundary layer thicknesses. However, in case of opposing buoyant force, a reverse mechanism regarding momentum and thermal boundary layer thicknesses was observed. The magnetic field intensity and Ohmic dissipation were directly proportional to each other. Hence, an increase in the intensity of magnetic field converts more electrical energy into heat (due to Ohmic dissipation process). It was also observed that an increase in the intensity of the magnetic field retarded the flow and reduced the momentum boundary thicknesses. Therefore, it is recommended applying an external magnetic field to control the flow and momentum boundary layer thickness. However, it is noted that an increase in the imposition of external magnetic field has opposite effect on the thermal boundary layer thicknesses due to Joule heating mechanism. It is also important to mention that momentum boundary layer thickness of hydrodynamic flow is higher than that of magnetohydrodynamic flow. However, thermal boundary layer thickness of hydrodynamic flow is less than that of magnetohydrodynamic flow. It was observed that the effective thermal conductivity of $\mathrm{Al}_{2} \mathrm{O}_{3}$ nano liquid was greater than that of $\mathrm{CuO}$ nanoliquid. Therefore, the application of $\mathrm{Al}_{2} \mathrm{O}_{3}$ is recommended if more transportation of heat is required. Convection on the surface plays a notable role in the temperature of nanoliquids ( $\mathrm{Al}_{2} \mathrm{O}_{3}$-liquid and $\mathrm{CuO}$-liquid). Further, heat generation in $\mathrm{CuO}$ liquid was less than that in 
$\mathrm{Al}_{2} \mathrm{O}_{3}$ in the vicinity of the wall. However, away from the wall, the opposite trend was observed.

A significant rise in the temperature due to an increase in intensity was observed. Therefore, control of Joule heating in the design of the thermal system is necessary. However, heat dissipation may be desirable in some biological fluid flows. Moreover, an increase in the intensity of the magnetic field caused an increase in the entropy generation. The positive buoyancy force enhanced the entropy generation. However, the opposing buoyancy force reduced energy losses. Energy losses in steady flow are higher than those in unsteady flow. The key observations are listed below:

- The buoyant force is responsible for the effect of thermal radiations on nanofluid flow. It is observed that if buoyant force is not considered, then there is no effect of thermal radiations on the flow and, hence, on momentum boundary layer thickness. Since the buoyant force is significant in vertical flows, it is recommended that horizontal arrangement of physical model (sheet) be made if no impact of thermal radiations on the flow of nanofluid is desired.

- The magnetic field decelerates the fluid motion due to hindrance caused by the Lorentz force. Therefore, it is recommended applying the external magnetic field perpendicular to the plane of sheet if momentum boundary layer thickness is to be controlled.

- Convectively heated surface causes greater entropy generation. Therefore, it is recommended not using the convectively heated surface.

- Imposition of external magnetic field increases the entropy generation and is responsible for great energy losses. Therefore, thermal systems work efficiently without losses of energy if external magnetic field is not imposed.

\section{Acknowledgement}

Authors Miss Shafia Rana and Dr. M. Nawaz are thankful to the Higher Education Commission (HEC) of Pakistan for the financial support under NRPU-vide No. 5855/Federal/NRPU/R \&D/HEC/2016.

\section{Nomenclature}

$u, v \quad$ Velocity components

Gr Grashof number

$\mathrm{Ha}$ Hartmann number

Ec Eckert number

$B_{i} \quad$ Biot number

$N_{r} \quad$ radiation parameter

Pr Prandtl number

$\begin{array}{ll}\operatorname{Re} & \text { Reynold number } \\ \mathrm{Br} & \text { Brinkman number } \\ N_{s} & \text { Entropy generation number } \\ \vec{q} & \text { radiative heat flux } \\ T & \text { Fluid temperature } \\ k & \text { Thermal conductivity } \\ \beta_{T} & \text { Volumetric expansion coefficient } \\ \kappa_{b} & \text { Boltzmann constant } \\ k^{*} & \text { Absorption coefficient } \\ \sigma^{*} & \text { Stefan-Boltzmann constant } \\ c_{p} & \text { Specific heat } \\ B_{0} & \text { Magnetic field } \\ Q & \text { Heat generation/absorption coefficient }\end{array}$

\section{Greek Symbols}

$\begin{array}{ll}\rho & \text { Density } \\ \sigma & \text { Electrical conductivity } \\ \varphi & \text { Volume fraction } \\ \mu & \text { Dynamic viscosity } \\ \eta & \text { Independent similarity function } \\ \lambda & \text { Unsteadiness parameter } \\ \Omega & \text { Temperature difference number }\end{array}$

\section{Subscripts}

$\begin{array}{ll}f & \text { Fluid } \\ n f & \text { Nanofluid } \\ \text { eff } & \text { Effective } \\ p & \text { Particles } \\ s & \text { Solid particles } \\ w & \text { Wall }\end{array}$

\section{References}

1. Einstein, A. "Eine neue Bestimmung der Molekuldimensionen", Ann. d. Phys., 19, pp. 289-306 (1906).

2. Brinkman, H.C. "The viscosity of concentrated suspensions and solutions", J. Chem. Phys., 20(4), pp. 571-581 (1952).

3. Batchelor G.K. "The effect of Brownian motion on the bulk stress in a suspension of spherical particles", $J$. Fluid Mech., 83(1), p. 97 (1977).

4. Mori, Y. and Ototake, N. "On the viscosity of suspensions", Chem. Eng., 20(9), pp. 488-494 (1956).

5. Wang, X., Xu, X., and Choi, S.U.S. "Thermal conductivity of nanoparticle-fluid mixture", J. Thermophys. Heat Transfer, 13(4), pp. 474-480 (1999).

6. Avsec, J. and Oblak, M. "The calculation of thermal conductivity, viscosity and thermodynamic properties 
for nanofluids on the basis of statistical nanomechanics", Int. J. Heat Mass Transfer, 50(21-22), pp. 43314341 (2007).

7. Masoumi, N. Sohrabi, N., and Behzadmehr, A. "A new model for calculating the effective viscosity of nanofluids", J. Phys. D Appl. Phys., 42(5), p. 55501 (2009).

8. Maxwell, J.C., A Treatise on Electricity and Magnetism, Oxford, Clarendon (1891).

9. Hamilton, R.L. and Crosser, O.K. "Thermal conductivity of heterogeneous two-component systems", I \& EC Fundamentals, 1, pp. 182-191 (1962).

10. Jeffrey, D.J. "Conduction through a random suspension of spheres", Proceedings of Royal Society, 335, pp. 355-367 (1973).

11. Bruggeman, D.A.G. "Berechnung verschiedener physikalischer konstanten von heterogenen substanzen", I. Dielektrizitatskonstanten und Leitfahigkeiten der Mischkorper aus Isotropen Substanzen. Annalen der Physik. Leipzig, 24, pp. 636-679 (1935).

12. Lu, S.Y. and Liu, H.C. "Effective conductivity of composites containing aligned spheroidal inclusions of finite conductivity", J. of Applied Physics, 79(9), pp. 6761-6769 (1996).

13. Davis, R.H. "The effective thermal conductivity of a composite material with spherical inclusions", Int. J. of Thermophysics, 7, pp. 609-620 (1986).

14. Sastry, N.N., Bhunia, V., Sundararajan, T., and Das, S.K. "Predicting the effective thermal conductivity of carbon nanotube based nanofluids", Nanotechnology, 19, p. 055704 (2008).

15. Syam Sunder, L.S., Singh, M.K., and Sousa, C.M.A. "Investigation of thermal conductivity and viscosity of $\mathrm{Fe}_{3} \mathrm{O}_{4}$ nanofluid for heat transfer applications", Int. Commun. In Heat and Mass Transfer, 44, pp. 7-14 (2013).

16. Xuan, Y., Li, Q., and Hu, W. "Aggregation structure and thermal conductivity of nanofluids", J. of American Institute of Chemical Engineers (AIChE), 49(4), pp. 1038-1043 (2003).

17. Jang, S.P. and Choi, S.U.S. "Role of Brownian motion in the enhanced thermal conductivity of nanofluids", Appli. Phy. Letters, 84, pp. 4316-4318 (2004).

18. Kumar, D.H., Patel, H.E., Kumar, V.R.R., Sundararajan, T., Pradeep, T., and Das, S.K. "Model for heat conduction in nanofluids", Physical Review Letters, 93(14), pp. 144301-1-144301-4 (2004).

19. Koo, J. and Kleinstreuer, C. "A new thermal conductivity model for nanofluids", J. of Nanoparticle Research, 6(6), pp. 577-588 (2004).

20. Bhattacharya, P., Phelan, P.E., and Prasher, R. "Brownian-motion-based convective conductive model for the effective thermal conductivity of nanofluids", J. of Heat Transfer, 128, pp. 588-595 (2006).
21. Xu, J., Yu, B., Zou, M., and Xu, P. "A new model for heat conduction of nanofluids based on fractal distributions of nanoparticles", J. of Appli. Phy., 39, pp. 4486-4490 (2006).

22. Yu-Hua, L., Wei, Q., and Ian-Chao, F. "Temperature dependence of thermal conductivity of nanofluids", Chinese Physics Letters, 25(9), p. 3319 (2008).

23. Shukla, R.K. and Dhir, V.K. "Effect of Brownian motion on thermal conductivity of nanofluids", J. of Heat Transfer, 130(4), pp. 042406-1-042406-13 (2008).

24. Yang, B. "Thermal conductivity equations based on Brownian motion in suspensions of nanoparticles (nanofluids)", J. of Heat Transfer, 130(4), pp. 0424081- 042408-5 (2008).

25. Sheikholeslami, M. "KKL correlation for simulation of nanofluid flow and heat transfer inapermeable channel", Physics Letters A., 1(137), pp. 1-9 (2014).

26. Sheikholeslami, M., Jafaryar, M., and Li, Z. "Second law analysis for nanofluid turbulent flow inside a circular duct in presence of twisted tape turbulators", J. of Molecular Liquids, 263, pp. 489-500 (2017).

27. Sheikholeslami, M. "Application of Darcy law for nanofluid flow in a porous cavity under the impact of Lorentz forces", J. of Molecular Liquids, 266, pp. 495-503 (2018).

28. Sheikholeslami, M. "Solidification of NEPCM under the effect of magnetic field in a porous thermal energy storage enclosure using nanoparticles", J. of Molecular Liquids, 263, pp. 303-315 (2018).

29. Sheikholeslami, M. and Rokni, H.B. "CVFEM for effect of Lorentz forces on nanofluid flow in a porous complex shaped enclosure by means of Nonequilibrium model", J. of Molecular Liquids, 254, pp. 446-462 (2018).

30. Sheikholeslami, M., Shehzad, S.A., Li, Z., and Shafee, A. "Numerical modeling for alumina nanofluid magnetohydrodynamic convective heat transfer in a permeable medium using Darcy law", Int. J. of Heat and Mass Transfer, 127, pp. 614-622 (2018).

31. Sheikholeslami, M., Li, Z., and Shafee, A. "Lorentz forces effect on NEPCM heat transfer during solidification in a porous energy storage system", Int. J. of Heat and Mass Transfer, 127, pp. 665-674 (2018).

32. Sheikholeslami, M. Jafaryar, M., Saleem, S., Li, Z., Shafee, A., and Jiang, Y. "Nanofluid heat transfer augmentation and exergy loss inside a pipe equipped with innovative turbulators", Int. J. of Heat and Mass Transfer, 126, pp. 156-163 (2018).

33. Sheikholeslami, M., Shehzad, S.A., and Li, Z. "Water based nanofluid free convection heat transfer in a three dimensional porous cavity with hot sphere obstacle in existence of Lorenz forces", Int. J. of Heat and Mass Transfer, 125, pp. 375-386 (2018).

34. Sheikholeslami, M., Darzi, M., and Li, Z. "Experimental investigation for entropy generation and energy loss of nano-refrigerant condensation process", Int. J. of Heat and Mass Transfer, 125, pp. 1087-1095 (2018). 
35. Sheikholeslami, M., Darzi, M., and Sadoughi, M.K. "Heat transfer improvement and pressure drop during condensation of refrigerant-based nanofluid; an experimental procedure", Int. J. of Heat and Mass Transfer, 122, pp. 643-650 (2018).

36. Sheikholeslami, M., Shehzad, S.A., Abbasi, F.M., and $\mathrm{Li}$, Z. "Nanofluid flow and forced convection heat transfer due to Lorentz forces in a porous lid driven cubic enclosure with hot obstacle", Comput. Methods Appl. Mech. Engrg., 338, pp. 491-505 (2018).

37. Sheikholeslami, M. "CuO-water nanofluid flow due to magnetic field inside a porous media considering Brownian motion", J. of Molecular Liquids, 249, pp. 921-929 (2018).

38. Sheikholeslami, M., Li, Z., and Shafee, A. "Lorentz forces effect on NEPCM heat transfer during solidification in a porous energy storage system", Int. J. of Heat and Mass Transfer, 127, pp. 665-674 (2018).

39. Malvandi, A., Safaei, M.R., Affash, M.H.K., and Ganji, D.D. "MHD mixed convection in a vertical annulus filled with $\mathrm{Al}_{2} \mathrm{O}_{3}$-water nanofluid considering nanoparticle migration", J. of Magnetism and Magnetic Materials, 382, pp. 296-306 (2015).

40. Domairry, G. and Hatami, M. "Squeezing Cu-water nanofluid flow analysis between parallel plates by DTM-Pade method", J. of Molecular Liquids, 193, pp. 37-44 (2014).

41. Alinia, M., Ganji, D.D., and Gorji-Bandpy, M. "Numerical study of mixed convection in an inclined two sided lid driven cavity filled with nanofluid using twophase mixture model", Int. Commun. in Heat and Mass Transfer, 38, pp. 1428-1435 (2011).

42. Hatami, M., Sheikholeslami, M., Hosseini, M., and Domiri Ganji, D. "Analytical investigation of MHD nanofluid flow in non-parallel walls", J. of Molecular Liquids, 194, pp. 251-259 (2014).

43. Ahmadi, A.R., Zahmatkesh, A., Hatami, M., and Ganji, D.D. "A comprehensive analysis of the flow and heat transfer for a nanofluid over an unsteady stretching flat plate", Powder Technology, 258, pp. 125-133 (2014).

44. Malvandi, A., Hedayati, F., and Domairry, G. "Stagnation point flow of a nanofluid toward an exponentially stretching sheet with nonuniform heat generation/absorption", J. of Thermodynamics, 2013, pp. 1-12 (2013).

45. Khorasanizadeh, H., Amani, J., and Nikfar, M. "Numerical investigation of $\mathrm{Cu}$-water nanofluid natural convection and entropy generation within a cavity with an embedded conductive baffle", Scientia Iranica, 19(6), pp. 1996-2003 (2012).

46. Sheikholeslami, M. and Gangi, D.D. "MHD flow in a permeable channel filled with nanofluid", Scientia Iranica, 21(1), pp. 203-212 (2014).

47. Hosseinzadeh, Kh., Afsharpanah, F., Zamani, S., Gholinia, M., and Ganji, D.D. "A numerical investigation on ethylene glycoltitanium dioxide nanofluid convective flow over a stretching sheet in presence of heat generation/absorption", Case Studies in Thermal Engineering, 12, pp. 228-236 (2018).

48. Ghadikolaei, S.S., Hosseinzadeh, Kh., Ganji, D.D., and Jafari, B. "Nonlinear thermal radiation effect on magneto Casson nanofluid flow with Joule heating effect over an inclined porous stretching sheet", Case Studies in Thermal Engineering, 12, pp. 176-187 (2018).

49. Amiri, A.J., Ardahaie, S.S., Amooie, A., Hosseinzadeh, Kh., and Ganji, D.D. "Investigating the effect of adding nanoparticles to the blood flow in presence of magnetic field in a porous blood arterial", Informatics in Medicine Unlocked, 10, pp. 71-81 (2017).

50. Ghadikolaei, S.S., Hosseinzadeh, Kh., Yassari, M., Sadeghi, H., and Ganji, D. D. "Boundary layer analysis of micropolar dusty fluid with $\mathrm{TiO}_{2}$ nanoparticles in a porous medium under the effect of magnetic field and thermal radiation over a stretching sheet", Journal of Molecular Liquids, 244, pp. 374-389 (2017).

51. Ghadikolaei, S.S., Hosseinzadeh, Kh., Hatami, M., and Ganji, D.D. "MHD boundary layer analysis for micropolar dusty fluid containing hybrid nanoparticles $\left(\mathrm{Cu}, \mathrm{Al}_{2} \mathrm{O}_{3}\right)$ over a porous medium", Journal of Molecular Liquids, 268, pp. 813-823 (2018).

52. Ghadikolaei, S.S., Hosseinzadeh, Kh., and Ganji, D.D. "Investigation on three dimensional squeezing flow of mixture base fluid (ethylene glycol-water) suspended by hybrid nanoparticle $\left(\mathrm{Fe}_{3} \mathrm{O}_{4}-\mathrm{Ag}\right)$ dependent on shape factor", Journal of Molecular Liquids, 262, pp. 376-388 (2018).

53. Ghadikolaei, S.S., Hosseinzadeh, Kh., Ganji, D.D., and Hatami, M. " $\mathrm{Fe}_{3} \mathrm{O}_{4}-\left(\mathrm{CH}_{2} \mathrm{OH}\right)^{2}$ nanofluid analysis in a porous medium under MHD radiative boundary layer and molecular dusty fluid", Journal of Molecular Liquids, 258, pp. 172-185 (2018).

54. Ghadikolaei, S.S., Hosseinzadeh, Kh., Hatami, M., Ganji, D.D., and Armin, M. "Investigation for squeezing flow of ethylene glycol $\left(\mathrm{C}_{2} \mathrm{H}_{6} \mathrm{O}_{2}\right)$ carbon nanotubes (CNTs) in rotating stretching channel with nonlinear thermal radiation", Journal of Molecular Liquids, 263, pp. 10-21 (2018).

55. Ghadikolaei, S.S., Hosseinzadeh, K.H., and Ganji, D.D. "MHD raviative boundary layer analysis of micropolar dusty fluid with graphene oxide (Go)-engine oil nanoparticles in a porous medium over a stretching sheet with joule heating effect", Powder Technology, 338, pp. 425-437 (2018).

56. Hosseinzadeh, Kh., Amiri, A.J., Ardahaie, S.S., and Ganji, D.D. "Effect of variable Lorentz forces on nanofluid flow in movable parallel plates utilizing analytical method", Case Studies in Thermal Engineering, 10, pp. 595-610 (2017).

57. Sheikholeslami, M., Hatami, M., and Domairry, G. "Numerical simulation of two phase unsteady nanofluid flow and heat transfer between parallel plates in presence of time dependent magnetic field", J. of the Taiwan Institute of Chemical Engineers, 46, pp. 43-50 (2015). 
58. Bejan, A. "Entropy generation minimization, the new thermodynamics of finite-size devices and finite-time processes", J. Appl. Phys., 79, pp. 1191-1218 (1996).

59. Bhatti, M.M., Rashidi, M.M., and Pop, I. "Entropy generation with nonlinear heat and mass transfer on MHD boundary layer over a moving surface using SLM", Nonlinear Eng., 6, pp. 43-52 (2017).

60. Armaghania, T., Kasaeipoora, A., Alavib, N., and Rashidic, M.M. "Numerical investigation of wateralumina nanofluid natural convection heat transfer and entropy generation in a based L-shaped cavity", $J$. Mol. Liq., 223, pp. 243-251 (2016).

61. Bianco, V., Nardini, S., and Manca, O. "Enhancement of heat transfer and entropy generation analysis of nanofluids turbulent convection flow in square section tubes", Nanoscale Research Letters, 6, p. 252 (2011).

62. Butt, A.S. and Ali, A.A. "Computational study of entropy generation in magnetohy-drodynamic flow and heat transfer over an unsteady stretching permeable sheet", The European Physical J. Plus, 129, pp. 1-13 (2014).

63. Das, S., Chakraborty, S., Jana, R.N., and Makinde, O.D. "Entropy analysis of unsteady magneto-nanofluid flow past accelerating stretching sheet with convective boundary condition", Appl. Math. Mech. -Engl. Ed., 36(12), pp. 1593-1610 (2015).

64. Abolbashari, M.H., Freidoonimehr, N., Nazari, F., and Rashidi, M.M. "Entropy analysis for an unsteady MHD flow past a stretching permeable surface in nanofluid", Powder Technology, 267, pp. 256-267 (2014).

\section{Biographies}

Shafia Rana is an MS student and is working as a Graduate Research Assistant (GRA) at Applied Mathematics \& Statistics, Institute of Space Technology, Islamabad, Pakistan. She did BS (Hons) in Mathematics (in 2016) at Government College and University (GCU), Lahore, Pakistan. His area of specialization is Computational Fluid Dynamics (CFD). She is working on HEC-funded project titled "Hall and ion slip effects on transport phenomena in the flows of nano-fluids" and has published more than 4 research articles on contents of this project. She is good in computer programing. She has used FEM and FDM methods as computing tools for the simulation of nano-fluids.

Muhammad Nawaz is working as an Associate Professor in Applied Mathematics at Institute of Space Technology Islamabad, Pakistan. He did $\mathrm{PhD}$ in Applied Mathematics (in 2012) from Quaid-e-Azam University (QAU), Islamabad, Pakistan. He earned his Master of Philosophy (MPhil) degree in Applied Mathematics in 2000 from Quaid-e-Azam University, Islamabad, Pakistan. Before this, he earned MSc degree from Bahauddin Zakarayia University (BZU), Multan, Pakistan. His area of specialization is Computational Fluid Dynamics (CFD) and, till now, he has published 45 research articles in international journals of very good repute. These published articles are on heat and mass transfer in Newtonian and nonNewtonian fluid flows using FEM, FVM, and analytical approximate methods. Recently, he has been working on the role of nano-fluids in heat transfer enhancement. He has supervised 15 MPhil students, and $3 \mathrm{PhD}$ students are working under his supervision. He has completed three R\&D projects and has won research productivity award 2012. His name is in the directory of productive scientists of the country.

Imran Haider Qureshi is a PhD scholar at Applied Mathematics \& Statistics, Institute of Space Technology, Islamabad, Pakistan. He did Master of Philosophy (MPhil) in Mathematics (in 2011) from Quaid-e-Azam University (QAU), Islamabad, Pakistan. He also earned the degree of Master of Science (MSc) in Mathematics from University of Gujrat, Gujrat, Pakistan. His area of interest is Computational Fluid Dynamics (CFD). He has published several research articles in international journals of very good repute. Using Finite Element Method (FEM), he is engaged in investigating thermo-physical properties of the Newtonian and nonNewtonian fluids. 